\title{
Niveles de fertilización inorgánica en tres variedades de kiwicha (Amaranthus caudatus L.) en Ancash, Perú
}

\author{
Inorganic fertilization levels in three varieties of kiwicha \\ (Amaranthus caudatus L.) in Ancash, Peru \\ Rhodes Leopoldo Mejía Valvas ${ }^{1}$, Luz Gómez Pando², Rember Emilio Pinedo Taco ${ }^{2 *}$, \\ Hugo Mendoza Vilcahuamán ${ }^{1}$, Clay Eusterio Pajuelo Roldán ${ }^{1}$
}

\section{RESUMEN}

La kiwicha (Amaranthus caudatus L.), considerada fuente alternativa de proteína de origen vegetal, es cultivada mayormente enzonas de temporal con bajos rendimientos. Por ello es importante desarrollar prácticas de manejo eficiente de los insumos y recursos como variedades y niveles de fertilización para incrementar su productividad. El estudio se realizó con el objetivo de determinar la interacción de dos variedades de kiwicha (Oscar Blanco, Centenario) y el ecotipo Caracino con tres niveles de fertilización (NPK), bajo un Diseño de Bloque Completo al Azar (DBCA) con un arreglo factorial $3_{\mathrm{V}}$ x $3_{\mathrm{F}}$ y cuatro repeticiones en condiciones de la localidad de Mato, provincia de Huaylas, Ancash. Las variables en estudio fueron altura de planta, días a la floración, días a la maduración, peso de mil granos, rendimiento y contenido de proteína. Con la dosis de 120-100-80 se hallaron los mayores valores para rendimiento, altura de planta y número de días a la maduración, con 2105,58 $\mathrm{kg} \mathrm{ha}^{-1}, 90,50 \mathrm{~cm}$ y 123,83 días, respectivamente, mientras que con la dosis 18-46-30 en peso de mil granos y contenido de proteína con 1,12 g y 20,19\%, respectivamente. La variedad Centenario alcanzó los mayores valores para rendimiento y peso de 1000 granos con $2104,75 \mathrm{~kg}^{-1}$ y 1,09 g, mientras que en contenido de proteína el ecotipo Caracino con 20,01\% resultó mayor respecto a Oscar Blanco (18,72\%) y Centenario $(18,35 \%)$.

Palabras clave: ecotipo, experimento, fertilización, interacción, localidad, potencial de rendimiento.

\begin{abstract}
Kiwicha (Amaranthus cuadatus L.) is an alternative source of protein of vegetable origin, grown mostly in temporary and with low yields, so it is important to develop efficient management practices of varieties and fertilization levels to increase their productivity. The study was conducted with the objective of determining the interaction of two varieties of kiwicha (Oscar Blanco, Centenario) and the Caracino ecotype with three levels of fertilization (NPK), under a Randomized Complete Block Design (DBCA) with a factorial arrangement $3_{V} \times 3_{F}$ and four repetitions in conditions of the town of Mato province of Huaylas, Ancash. The variables under study were: plant height, days of flowering, and days of maturation, weight of a thousand grains, yield and protein content. With the dose of 120-100-80 the highest values were found for yield, plant height and number of days at maturation, with $2105.58 \mathrm{~kg} \mathrm{ha}-1,90.50 \mathrm{~cm}$ and 123.83 days, respectively; while with the dose 18-46-30 in weight of one thousand grains and protein content with $1.12 \mathrm{~g}$ and $20.19 \%$, respectively. The Centennial variety resulted in the highest values for yield and weight of 1000 grains with 2104.75 and $1.09 \mathrm{~g}$; while in protein content the Caracino ecotype with $20.01 \%$ was higher compared to Oscar Blanco (18.72\%) and Centenario (18.35\%).
\end{abstract}

Keywords: ecotype, experiment, fertilization, interaction, location, yield potential.

\section{Introducción}

El amaranto, millmi, coimi o kiwicha (Amaranthus caudatus L.) se encuentra cultivado en Sudamérica desde Colombia hasta el norte de Argentina (Rojas et al., 2010), es decir, en toda la zona andina. La kiwicha es originaria de los Andes, como especie de día corto más adaptada a las bajas temperaturas que las otras especies de amaranto (Jiménez et al., 2018; Mujica y Berti, 1997; Roque, 2019; Rojas et al., 2010; Tapia, 2000). Su amplia diversidad genética y plasticidad

1 Universidad Nacional Santiago Antúnez de Mayolo (UNASAM), Escuela Profesional de Agronomía Ancash, Perú

2 Universidad Nacional Agraria La Molina (UNALM). Facultad de Agronomía, Departamento de Fitotecnia, Lima, Perú.

* Autor para correspondencia: rpinedo@lamolina.edu.pe

Fecha de Recepción: 15 de Octubre, 2019.

Fecha de Aceptación: 8 de Diciembre, 2019. 
fenotípica le permiten adaptarse a condiciones desfavorables de temperatura y humedad (Rojas et al., 2010). Su crecimiento rápido y su rusticidad para prosperar en suelos pobres le confieren un alto potencial agronómico (Andrade y Simbaña, 2013; Ramírez et al., 2011; Tapia, 2000; Topwal, 2019). Complementariamente, su capacidad para tolerar temperaturas altas contribuye a la gran adaptabilidad geográfica del cultivo a numerosas condiciones ambientales (de Troiani et al., 2005; Topwal, 2019).

Las especies de amaranto se adaptan bien a las condiciones de la zona agroecológica Quechua (2700 a $3200 \mathrm{msnm}$ ), en climas templados con temperaturas entre $15-20^{\circ} \mathrm{C}$ y con precipitaciones no menores de $600 \mathrm{~mm}$ (Tapia, 2000). Sin embargo, debido a su plasticidad genética se puede cultivar desde el nivel del mar hasta los $3000 \mathrm{msnm}$ (Rojas et al., 2010). En cuanto a suelos, se adapta bien a suelos francos de buen drenaje y soporta un $\mathrm{pH}$ del suelo desde 6,2 hasta 7,8 y con cierta tolerancia a condiciones salinas. El amaranto se cultiva principalmente en los valles interandinos de la sierra y en pequeñas extensiones; mayormente en siembras asociadas a maíz o formando bordes en otros cultivos (Andrade y Simbaña, 2013; Mujica y Berty, 1997; Tapia, 2000).

La superficie cultivada en el Perú en el año 2017 alcanzó a 1428 ha, con un rendimiento promedio nacional de $1885 \mathrm{~kg} \mathrm{ha}^{-1}$. A nivel departamental, Ancash después de Arequipa ocupa el segundo lugar con 162 ha. En las zonas de valles interandinos similares al ámbito de estudio, el cultivo se practica en parcelas de pequeña y mediana escala, predominantemente en superficies menores a una hectárea, empleando tecnologías de producción tradicional y algunas en proceso de transición a sistemas de producción convencional, pero con bajos niveles de productividad y rentabilidad (Barreto et al., 2015; Pinedo et al., 2018).

La fertilización de la kiwicha con productos orgánicos e inorgánicos es una práctica necesaria para conseguir altos rendimientos y productos de calidad, especialmente para la producción destinada al mercado nacional e internacional (Ramírez et al., 2011). Sin embargo, en los sistemas de producción tradicionales orientados al autoconsumo, normalmente no se aplican fertilizantes; por lo que los rendimientos son relativamente bajos (Barreto et al., 2015; Pinedo et al., 2018).

El amaranto es un cultivo temporal y de secano, por lo cual la aplicación de fertilizantes debe estar estrechamente relacionada con la disponibilidad de humedad. Por ello, los requerimientos nutricionales estarán definidos por las condiciones ambientales de la zona donde sea cultivado (Hernández et al., 2014; Ramírez et al., 2011). En condiciones adecuadas de suelos (neutros o básicos), humedad y temperatura, produce hasta $5000 \mathrm{~kg} \mathrm{ha}^{-1}$; aunque en promedio se obtienen rendimientos de 1000 a $2500 \mathrm{~kg} \mathrm{ha}^{-1}$ (Mujica y Berti, 1997).

Por lo tanto, el rendimiento de granos y la calidad en los cultivos está relacionado con la cantidad de elementos nutricionales que son absorbidos durante el ciclo de cultivo y el efecto de otros factores del suelo, el clima y el genotipo. Se considera que el amaranto es exigente en nutrientes. Por ejemplo, el déficit de nitrógeno se manifiesta con prontitud en las plantas, que muestran amarillamiento, retraso en el crecimiento y emergencia prematura de la panoja, lo que trae como consecuencia baja producción (Estrada, 2011). Con respecto al uso de los fertilizantes en el cultivo de amaranto con fuentes nitrogenadas, fosforadas y potásicas, estas sustancias tienen efectos positivos en la producción de granos y el contenido de proteínas (Mujica y Berty, 1997; Ramírez et al., 2011; Tapia, 2000). Sin embargo, los estudios sobre el efecto de la fertilización potásica son escasos (de Troiani et al., 2005). Al respecto, Romero et al. (2017), con el nivel 8020-00 obtuvieron el mejor rendimiento de grano por planta.

En general el amaranto requiere dosis medias de nitrógeno y fósforo, y es menos necesaria la aplicación del potasio sobre todo en el área andina, pese a que el $\mathrm{K}$ activa más de 60 enzimas y juega un papel vital en la síntesis de carbohidratos y proteínas (Pinedo et al., 2017). Pero no es así en el área costera del Perú, donde con las dosis de 200-240 de N, 100 a $150 \mathrm{~kg}$ de $\mathrm{P}_{2} \mathrm{O}_{5}$ y 50 a $80 \mathrm{~kg} \mathrm{~K}_{2} \mathrm{O}$ el cultivo rinde de 4 a $5 \mathrm{t} \mathrm{ha}^{-1}$ (Mujica y Berty, 1997; Tapia, 2000). En el área andina, generalmente corresponde a $80 \mathrm{~kg} \mathrm{~N} / \mathrm{ha}$, $60 \mathrm{~kg} \mathrm{P}_{2} \mathrm{O}_{5}$ y 0 o $40 \mathrm{~kg} \mathrm{ha}^{-1}$ de $\mathrm{K} 2 \mathrm{O}$ (Mujica y Berty 1997). Sin embargo, dosis bajas de NPK no siempre resultan con rendimiento aceptables, tal como reportan Ramírez et al. (2011), quienes empleando las fórmulas 80-60-40 y 80-30-40 hallaron rendimientos de 668,7 y $1660,9 \mathrm{~kg} \mathrm{ha}^{-1}$. Por lo tanto, la productividad del cultivo se puede atribuir a otros factores como el ambiente. Tapia (2000) destaca que en ensayos realizados por 
el INIA en Ayacucho con el nivel 120-80-20 se alcanzaron $1889 \mathrm{~kg} \mathrm{ha}^{-1}$, mientras que en la costa, con la fórmula 240-150-80 se obtuvieron 4,5 $\mathrm{t} \mathrm{ha}^{-1}$ (Irrigación Majes, Arequipa, Perú).

Se puede aseverar que las especies cultivadas de amaranto responden bastante bien a niveles elevados de nitrógeno, que también influye en el rendimiento (Romero et al., 2017). Finalmente, la aplicación de fertilizantes y el comportamiento de la variedad pueden influir en el contenido de proteína. Con respecto a la composición química de la semilla de amaranto (por $100 \mathrm{~g}$ de parte comestible y en base seca), se encuentra en un rango de $12 \%$ a $19 \%$ (Topwal, 2019).

Por lo expuesto, y debido a que el paquete tecnológico para la producción de amaranto no está totalmente definido, se realizó este experimento con el objetivo de determinar los efectos de la fertilización en variedades comerciales y un ecotipo local de kiwicha este.

\section{Materiales y métodos}

El experimento se realizó desde diciembre de 2017 hasta mayo de 2018, en el distrito de Mato (Figura 1), ubicado a $2735 \mathrm{msnm}$ en la provincia de Yungay, región Ancash (Latitud Sur 12 ${ }^{\circ}$ 56' 22.8" y Latitud Oeste 74 14' 51.5").

Según el análisis químico y físico y caracterización del suelo, el suelo es ligeramente alcalino con un de $\mathrm{pH}$, bajo en materia orgánica $(1,66 \%)$, contenido medio en $\mathrm{P}$ (10,2 ppm), medio en $\mathrm{K}$ (146 ppm), textura con 55\%, $24 \%$ y $18 \%$ de arena, limo y arcilla, respectivamente. De acuerdo con Soto (2010), el amaranto prefiere suelos francos, arenosos, con buen contenido de materia orgánica y con buen drenaje, $\mathrm{pH}$ de 6 a 7,5.

Como material genético se evaluaron un ecotipo local y dos variedades mejoradas de kiwicha. Caracino es un ecotipo local colectado en la zona de estudio. Con respecto a las variedades comerciales, Oscar Blanco es una variedad de grano blanco mejorada, obtenida por selección en el Programa de Investigación en Kiwicha del Centro de Investigación en Cultivos Andinos de la UNSAAC en el año 1982, y la variedad Centenario es de grano grande, blanco cremoso, liberada por el Programa de Investigación y Proyección Social de Cereales y Granos Nativos de la Universidad Nacional Agraria La Molina el año 2006. Las fuentes de fertilizantes utilizadas fueron urea (46\% de $\mathrm{N}$ ), fostato diamónico (18 de $\mathrm{N}$ y $46 \%$ $\left.\mathrm{P}_{2} \mathrm{O}_{5}\right)$ y cloruro de potasio $\left(60 \%\right.$ de $\left.\mathrm{K}_{2} \mathrm{O}\right)$ (Tabla 1$)$.

Para el experimento se empleó un Diseño Completamente al Azar (DBCA) con arreglo factorial de 3 x 3 con cuatro repeticiones. Los tratamientos resultan de las combinaciones de los factores en estudio. Para la comparación de medias se utilizó la prueba de Rango Múltiple de Duncan con nivel de significación de $5 \%$.

La fase experimental duró 150 días a partir de la siembra, que se realizó a chorro continuo siguiendo la línea del surco (Soto, 2010; Jiménez et al., 2018). Por la época de siembra se hicieron riegos con una frecuencia de 15 días hasta la etapa de madurez fisiológica del cultivo. Se evaluaron las siguientes variables: Días a la Floración Media (DFME =

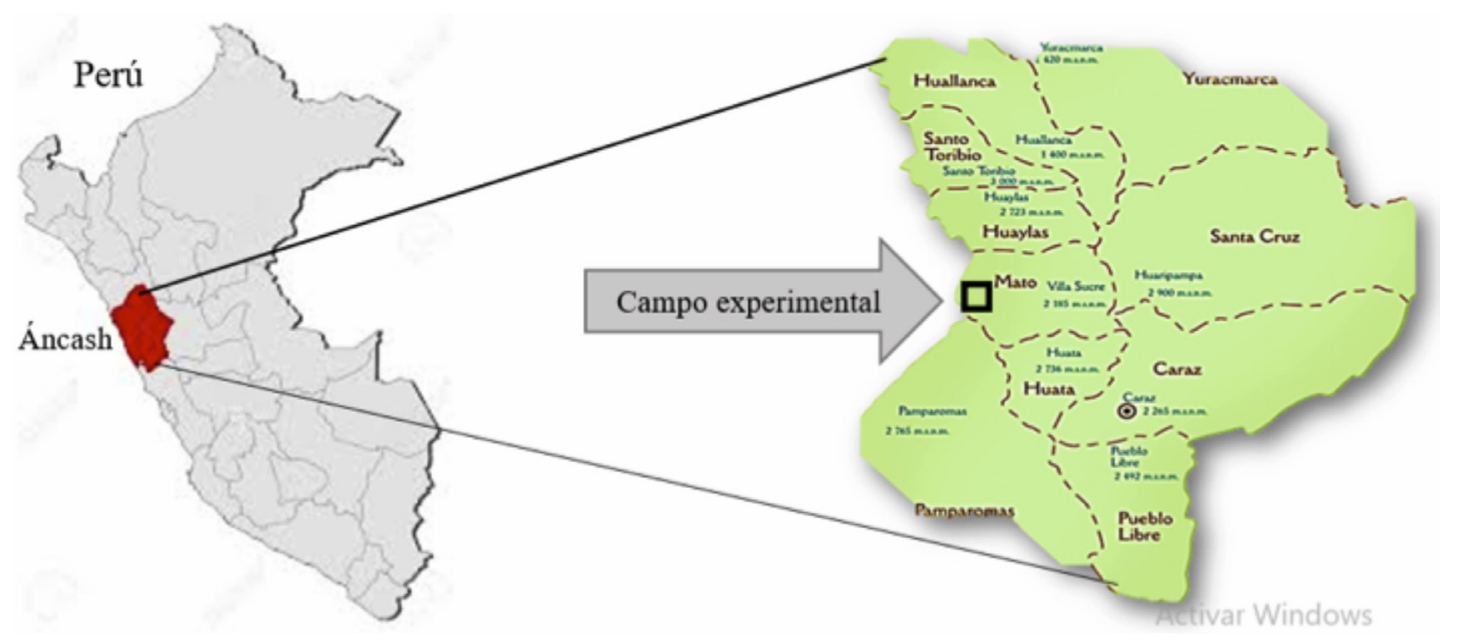

Figura 1. Ubicación del campo experimental, distrito de Mato, Provincia de Huaylas, departamento de Áncash, Perú 
Tabla 1. Variedades y niveles de fertilización para ensayo de niveles de fertilización y variedades de kiwicha.

\begin{tabular}{clllrrrrr}
\hline \multicolumn{2}{c}{ Variedades de kiwicha } & & \multicolumn{3}{c}{ Niveles de fertilización } & & \multirow{2}{*}{ Observaciones } \\
\cline { 5 - 6 } Clave variedades & Variedades & & & Clave Niveles & $\mathrm{N}$ & $\mathrm{P}_{2} \mathrm{O}_{5}$ & $\mathrm{~K}_{2} \mathrm{O}$ & \\
\hline v1 & Oscar Blanco & & $\mathrm{F}^{*}$ & 18 & 46 & 30 & & Testigo local \\
v2 & Centenario & & $\mathrm{F} 2$ & 100 & 80 & 60 & & Nivel medio \\
v3 & Caracino & & $\mathrm{F} 3$ & 120 & 100 & 80 & & Nivel alto \\
\hline
\end{tabular}

$\mathrm{F}_{1} *$ = Dosis testigo de fertilización (fertilización local).

número de días transcurridos desde la siembra hasta que el $50 \%$ de la población presentó antesis o liberación de polen) (Arellano y Galicia, 2007); Días a la Altura de Planta (ALPT = distancia en centímetros del suelo a la punta de la inflorescencia); Maduración y Rendimiento $(\mathrm{REND}=$ considerado como el peso de grano limpio al 10\% de humedad y expresado en $\mathrm{kg} \mathrm{ha}^{-1}$. En el estudio también se evaluaron variables de calidad como el Peso de Mil granos $(\mathrm{PMIL}=$ peso en $\mathrm{g}$ de dicha cantidad de semillas) y el contenido de proteínas de los granos expresado en porcentaje (\%) (Ortiz, 2018).

Cada unidad experimental constó de una parcela de dos metros de ancho por cinco metros de largo, en la que se dispusieron cuatro surcos separados 0,5 metros entre sí. En cada surco se sembró semilla de amaranto a chorro continuo. Se evaluaron las variables de los dos surcos centrales y el resto se dejó para evitar el efecto de borde (Andrade y Simbaña, 2013).

\section{Resultados y discusión}

\section{Variables agronómicas}

En la Tabla 2 se presenta el ANOVA combinado para altura de planta, rendimiento, días a la floración y días a la maduración. Para las características evaluadas se observa que no existen diferencias significativas ( $\alpha=0.05$ ) para niveles de fertilización, repeticiones, variedades y la interacción niveles de fertilización por variedades. Los coeficientes de variación para altura de planta, rendimiento, días a la floración y días a la maduración fueron $7,70 \%, 16,41 \%, 4,99 \%$ y $3,98 \%$, respectivamente. Bajo condiciones térmicas del lugar del experimento, los cultivares en estudio alcanzaron la madurez entre los 122,83, y 123.58 días después de la siembra. Bajo condiciones térmicas de Ayacucho la variedad Oscar Blanco presenta un rango de precocidad comprendido entre 124 y 134 días, y Centenario se muestra tardía requiriendo un rango de 135 a 145 días (Roque, 2019). Según Soto (2010), en general los granos de kiwicha alcanzan su madurez entre 130 y 180 días.

\section{Altura de planta (cm)}

En la Tabla 3, la ALPT en las tres dosis de fertilización estudiadas varió de 88,58 a 90,50 $\mathrm{cm}$. El valor más bajo corresponde a la dosis 18-46-30 y el más alto a la dosis 120-100-80. Estos valores, en promedio de tres variedades, no difieren estadísticamente de acuerdo a la prueba Duncan $(\alpha=0,05)$.

Tabla 2. Cuadrados medios, significancias y coeficientes de variación (CV) de altura de planta $(\mathrm{cm})$, rendimiento, días a la floración y días a la maduración. Mato-Yungay. Campaña 2017-2018.

\begin{tabular}{lccrrr}
\hline \multirow{2}{*}{ Variables } & \multicolumn{4}{c}{ Cuadrados Medios } & CV \% \\
\cline { 2 - 5 } & Nivel $(\mathrm{N})$ & \multicolumn{1}{c}{ Variedad $(\mathrm{V})$} & $\mathrm{N} \mathrm{x} \mathrm{V}$ & \multicolumn{1}{c}{ Error } & \\
\hline ALPT & 11,08 & 9 & 66,33 & 47,54 & 5,19 \\
DAFM & 30,53 & 78,69 & 54,13 & 37,72 & 4,99 \\
DAM & 7 & 3,25 & 137,75 & 23,98 & 3,98 \\
RDTO & $95.560,11$ & $93.799,19$ & $91.795,36$ & $11.2661,9$ & 16,41 \\
\hline
\end{tabular}

**: Significativo con $\mathrm{P} \leq 0,01, *$ : significativo con $\mathrm{P} \leq 0,05$, ns: no significativo, $\mathrm{CV}$ : coeficiente de variación, ALPT: altura de planta, DAFM: días a floración masculina, DAMD: días a la maduración, REND: rendimiento de grano, APTA: altura de planta. 
Con respecto a ALPT en promedio de tres dosis de fertilización (Tabla 4), la prueba Duncan $(\alpha=0,05)$ muestra que no existen diferencias significativas en las tres variedades estudiadas. La variedad local Caracino alcanzó la mayor altura de $90,5 \mathrm{~cm}$, seguida por Centenario con $89,9 \mathrm{~cm}$ y Oscar Blanco con 88,3 cm. Según Soto (2010), la planta de kiwicha puede alcanzar hasta $\operatorname{los} 150 \mathrm{~cm}$ de altura de no mediar inconvenientes en su manejo. Se considera que el nitrógeno es el elemento más restrictivo en el crecimiento de un cultivo, y en el caso del amaranto se menciona que a medida que el ambiente sea más favorable se deberá aplicar mayor cantidad. Por ejemplo, Arellano y Galicia (2007) señalan que con las dosis de 80 y $160 \mathrm{~kg}$ de $\mathrm{N} \mathrm{ha}^{-1}$ la altura de planta fue significativamente mayor que con el tratamiento sin nitrógeno y hubo menor incidencia de plantas enfermas. Sin embargo, Romero et al. (2017) afirman que el N no tiene mucho efecto en la altura de planta.

En cuanto a la altura de planta, la variedad local Caracino alcanzó la mejor altura $(90,8 \mathrm{~cm})$, seguida por Oscar Blanco $(90,08 \mathrm{~cm})$ y Centenario $(89,58 \mathrm{~cm})$.

\section{Rendimiento de granos}

El mejor rendimiento de granos se observa con la dosis de 120-100-80 y el valor más bajo a la dosis de 18-46-30 (Tabla 3), valores determinados en promedio de variedades. Estos valores no difieren estadísticamente de acuerdo a la prueba Duncan $(\alpha=0,05)$. Se puede inferir que a dosis crecientes de fertilizantes se incrementa el rendimiento. En general la planta requiere cantidades de $\mathrm{N}$ entre 20 y $80 \mathrm{~kg} \mathrm{ha}^{-1}$, de $\mathrm{P}$ entre 00 y $40 \mathrm{~kg} \mathrm{ha}^{-1}$ y de 1 a $3 \mathrm{t} \mathrm{ha}^{-1}$ de compost de lombriz (Romero et al., 2017). La fórmula de fertilización $80-20-00 \mathrm{~kg}$ $\mathrm{ha}^{-1}$ de NPK más una tonelada de composta $\mathrm{ha}^{-1}$ fue la que obtuvo el mayor rendimiento de grano, equivalente a 2018,1 $\mathrm{kg} \mathrm{ha}^{-1}$. En cambio,
Ramírez et al. (2011), en el estado de Montecillo, México, hallaron los rendimientos más altos con las fórmulas 80-60-40 y 80-30-40 con 1668,7 y $1660,9 \mathrm{~kg} \mathrm{ha}^{-1}$, respectivamente. El cultivo responde a la fertilización nitrogenada (altas dosis). Una dosis de $180 \mathrm{~kg} /$ ha puede incrementar hasta $42 \%$ el rendimiento, pero el exceso puede provocar acame (Praveen y Usha, 2014).

El rendimiento de las variedades, en promedio de tres dosis de fertilización, con la prueba Duncan $(\alpha=0,05)$ muestra que no existen diferencias significativas para rendimiento de granos entre las tres variedades estudiadas (Tabla 4). Centenario presenta el mejor rendimiento de granos de $2104,75 \mathrm{~kg} \mathrm{ha}^{-1}$, seguida por Oscar Blanco con $2088,17 \mathrm{~kg} \mathrm{ha}^{-1}$ y la variedad local Caracino con $1944 \mathrm{~kg} \mathrm{ha}^{-1}$.

El INIA para la zona andina recomienda el nivel de 80-60-40 de NPK (Estrada, 2011), mientras que Peralta et al. (2012) sugieren la dosis 100-60-20, Otros resultados indican que el incremento de la dosis de nitrógeno redunda en una mayor productividad (Estrada, 2011). Al respecto, Ramírez et al. (2011) mencionan que las variedades Oscar Blanco y Centenario pueden alcanzar de 3 a 5 t ha $^{-1}$ en condiciones de manejo y fertilización ideales.

En el cultivo de amaranto es posible mejorar la productividad con un buen desarrollo de las plantas en condiciones de temporal, lo que depende en gran medida de la fertilidad de los suelos y la respuesta fisiológica de las especies (Hernández et al., 2014; Rojas et al., 2010). En un ensayo realizado en cinco localidades por Ortiz et al. (2018), se reportaron rendimientos desde 1832 hasta $3439 \mathrm{~kg} \mathrm{ha}^{-1}$ con una dosis de 80-40-0 kg de NPK.

\section{Días a la floración media (DFME)}

En la Tabla 3 se presentan los datos observados para DFME en las tres dosis de fertilización

Tabla 3. Medias de altura de planta $(\mathrm{cm})$, rendimiento $\left(\mathrm{kg} \mathrm{ha}^{-1}\right)$, días a la floración y días a la maduración de niveles de fertilización en promedio variedades. Mato, Yungay. Campaña 2017-2018.

\begin{tabular}{ccccc}
\hline NPK (fertilización) & Altura planta $(\mathrm{cm})$ & Rendimiento $\left(\mathrm{kg} \mathrm{ha}^{-1}\right)$ & Floración (días) & Maduración (días) \\
\hline $18-46-30$ & $88,58 \mathrm{a}$ & $1.943,08 \mathrm{a}$ & $78,08 \mathrm{a}$ & $122,33 \mathrm{a}$ \\
$100-80-60$ & $89,67 \mathrm{a}$ & $2.088,25 \mathrm{a}$ & $77,25 \mathrm{a}$ & $122,83 \mathrm{a}$ \\
$120-100-80$ & $90,50 \mathrm{a}$ & $2.105,58 \mathrm{a}$ & $75,00 \mathrm{a}$ & $123,83 \mathrm{a}$ \\
\hline
\end{tabular}

Medias con una letra común no son significativamente diferentes ( $p>0,05)$. 
estudiadas y variaron de 75,1 a 80,1 días. El valor más bajo corresponde a la dosis 120-100-80 de NPK y el más alto a la dosis 100-80-60 de NPK. Estos valores, en promedio de variedades, no difieren estadísticamente de acuerdo a la prueba Duncan $(\alpha=0,05)$. Al respecto, Arellano y Galicia (2007) indican que las características DFME no se modifican significativamente por efecto de las dosis de $\mathrm{N}(0-80-100 \mathrm{~N}) 90$ y en promedio este carácter se alcanza a los 102 días.

Los datos de DFME de las variedades, en promedio de tres dosis de fertilización, se presentan en la Tabla 4. La prueba Duncan $(\alpha=0,05)$ muestra que no existen diferencias significativas en días a la floración en las tres variedades estudiadas. La variedad local Caracino alcanzó DFME a los 74,42 días, seguida por Centenario con 76,42 y Oscar Blanco con 79,50 días.

\section{Días a la maduración (DMAD)}

Los DMAD variaron de 120,8 a 124,6 días, correspondiendo el valor más bajo a la dosis de 120-100-80 de NPK y el más alto a la dosis de 18-46-30 de NPK (Tabla 3), valores determinados en promedio de variedades. Estos valores no difieren estadísticamente, de acuerdo a la prueba Duncan $(\alpha=0,05)$.
En la Tabla 4, con respecto a la variable días a la maduración de las variedades, en promedio de tres dosis de fertilización, la prueba Duncan $(\alpha=0,05)$ muestra que no existen diferencias significativas para días a la maduración de las tres variedades estudiadas. Centenario fue la más precoz con 122,58 días, seguida por la variedad local Caracino con 122,58 días y Oscar Blanco como la más tardía con 123,58 días.

\section{Variables de calidad}

En la Tabla 5 se observa el ANOVA combinado para peso de 1000 granos y contenido de proteína del grano. Para las características evaluadas no se observaron diferencias significativas $(\alpha=0,05)$ en niveles de fertilización, repeticiones, variedades y la interacción niveles de fertilización $\mathrm{x}$ variedades. Los coeficientes de variación para peso de 1000 granos y contenido de proteína del grano fueron iguales a $10,75 \%$ y $15,63 \%$, respectivamente. La no significancia en la interacción indica que cada factor actuó independientemente (Pinedo et al., 2017).

\section{Peso de 1000 granos (g)}

En la Tabla 6 se presentan los datos observados en las tres dosis de fertilización estudiadas. El

Tabla 4. Medias de altura de planta $(\mathrm{m})$, rendimiento $\left(\mathrm{kg} \mathrm{ha}^{-1}\right)$, días a la floración y días a la maduración de variedades en promedio de fertilización. Mato, Yungay. Campaña 2017-2018.

\begin{tabular}{lcccc}
\hline \multicolumn{1}{c}{ Variedad } & Altura planta $(\mathrm{cm})$ & Rendimiento $\left(\mathrm{kg} \mathrm{ha}^{-1}\right)$ & Floración (días) & Maduración (días) \\
\hline Oscar Blanco & $90,08 \mathrm{a}$ & $2.088,17 \mathrm{a}$ & $79,50 \mathrm{a}$ & $123,58 \mathrm{a}$ \\
Centenario & $89,58 \mathrm{a}$ & $2.104,75 \mathrm{a}$ & $76,42 \mathrm{a}$ & $122,58 \mathrm{a}$ \\
Caracino & $90,8 \mathrm{a}$ & $1.944,00 \mathrm{a}$ & $74,42 \mathrm{a}$ & $122,83 \mathrm{a}$ \\
\hline
\end{tabular}

Medias con una letra común no son significativamente diferentes ( $p>0,05)$.

Tabla 5. Cuadrados medios, significancias y coeficientes de variación (CV) para peso de 1000 granos (g) y contenido de proteínas (\%) de granos.

\begin{tabular}{lccccc}
\hline \multirow{2}{*}{ Variables } & \multicolumn{4}{c}{ Cuadrados Medios } & \multirow{2}{*}{ CV \% } \\
\cline { 2 - 4 } & Nivel (N) & Variedad (V) & N x V & Error & \\
\hline PMGR & 0,01 & 0,0022 & 0,01 & 0,01 & 10,75 \\
CPRO & 13,49 & 9,16 & 1,90 & 8,84 & 15,63 \\
\hline
\end{tabular}

$* *$ : Significativo con $\mathrm{P} \leq 0,01, *$ : significativo con $\mathrm{P} \leq 0,05$, ns: no significativo. PMGR: Peso de mil granos, CPRO: contenido de proteína, CV: coeficiente de variación. 
rango promedio para peso de mil granos varió de $1,06 \mathrm{~g}$ a 1,11 g. El valor más bajo corresponde a la dosis 100-80-60 de NPK y el más alto a la dosis 18-46-30 de NPK. Estos valores, en promedio de tres variedades, no difieren estadísticamente de acuerdo a la prueba Duncan $(\alpha=0,05)$.

Con respecto a la variable peso de 1000 granos de las variedades, en promedio de dosis de fertilización, la prueba Duncan $(\alpha=0,05)$ muestra que no existen diferencias significativas. La variedad Centenario presentó el mayor peso con 1,09 g, seguida por Oscar Blanco con 1,8 g y la variedad local Caracino con 1,06 g. Este parámetro normalmente se utiliza como un indicador de rendimiento potencial de la harina refinada. Díaz-Ortega et al. (2004) afirman que el $\mathrm{N}$ no afecta el peso de mil semillas (PMS), y llegaron a esta conclusión luego de haber ensayado tres dosis de fertilización nitrogenada $(0,100 \mathrm{y}$ $200 \mathrm{~kg} \mathrm{ha}^{-1}$ de N).

\section{Contenido de proteína del grano (\%)}

El contenido de proteína fluctuó entre $18,11 \%$ y $20,19 \%$, correspondiendo el valor más bajo a la dosis de 100-80-60 de NPK y el más alto a la dosis de 120-100-80 de NPK (Tabla 6), valores promedio de tres variedades. Estos valores no difieren estadísticamente de acuerdo a la prueba Duncan $(\alpha=0,05)$.

En la Figura 2A se presentan los datos de contenido de proteína de granos de las variedades, en promedio de tres dosis de fertilización. La prueba Duncan $(\alpha=0,05)$ muestra que no existen diferencias significativas para esta característica en las tres variedades estudiadas. La variedad local Caracino tuvo el mayor valor con $20,01 \%$, seguida por Oscar Blanco con 18,72 \% y Centenario con 18,35\% (Figura 2B). Los estudios indican que la kiwicha es una buena fuente de proteínas (13\% a $19 \%$ ) con un adecuado balance de aminoácidos esenciales (Ayala et al., 2016; Paucar et al., 2017; Castro et al., 2012, Rojas et al., 2010; Topwal, 2019) y la cantidad de nutrientes puede ser influenciada por la variedad, la región en la cual se cultiva y las prácticas agronómicas y de fertilización que se le aplican (Hernández et al., 2014; Rojas et al., 2010). Sin embargo, algunos reportes indican que el contenido de nutrientes no presentó diferencias significativas entre las plantas fertilizadas y sin fertilizar (de Troiani et al., 2005).

El ecotipo Caracino con 20,01\% de proteína tiene el mayor valor con respecto a las dos variedades mejoradas, lo cual demuestra que las variedades nativas o locales poseen un enorme potencial, pero a falta de investigación y asistencia técnica son desplazadas fácilmente por variedades mejoradas introducidas (Ayala et al., 2016).

\section{Conclusiones}

No se observaron diferencias significativas en los valores obtenidos para las características evaluadas a nivel de promedio de las dosis de fertilización y de variedades, con excepción de la variantew peso de 1000 granos para variedades en promedio de nivel de fertilización.

En promedio de variedades, en la dosis de 120100-80 de NPK se encontraron los mayores valores para rendimiento igual a $2105,58 \mathrm{~kg} \mathrm{ha}^{-1}$, altura de planta $90.50 \mathrm{~cm}$, para número de días a la maduración igual a 123,83 días y contenido de proteína con $19,86 \%$. En la dosis de 18-46-30 se observó el mayor valor de peso de 1000 granos en gramos.

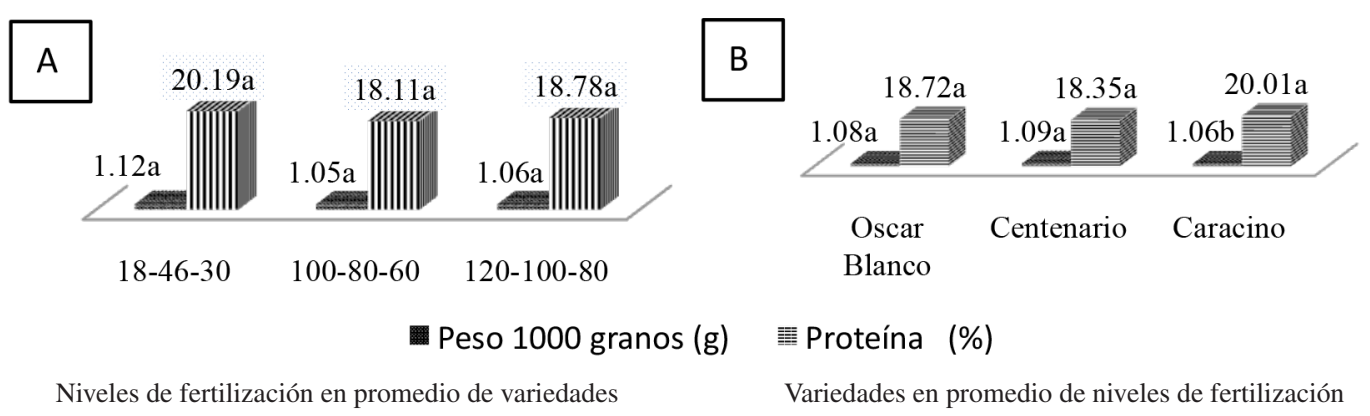

Figura 2. Promedios de las variables evaluadas en variedades y niveles de fertilización en variedades y ecotipo local de kiwicha en Mato, Yungay, 2017-2019. Medias con letras iguales en la misma columna no son estadísticamente diferentes (Duncan 0,05\%). 
En promedio de las dosis de fertilización destaca la variedad Centenario con 2104,75 kg $\mathrm{ha}^{-1}$ como el mayor rendimiento y con $1,09 \mathrm{~g}$ para peso de 1000 granos. En cambio, la variedad Oscar Blanco alcanzó los mayores valores con
79,50 para días a la floración y 123,58 para días a la maduración. La variedad local Caracino resultó con el mayor promedio de altura de planta con $90,80 \mathrm{~cm}$ y el mayor contenido de proteína con $20,01 \%$.

\section{Literatura Citada}

Andrade, V.; Simbaña, A.

2013. Manejo agronómico del amaranto y quinua con Metarhizium anisopliae para el control de insectos. AXIOMA, 2 (11): 5-11.

Arellano, J.L.; Galicia, J.A.

2007. Rendimiento y características de planta y panoja de amaranto en respuesta a nitrógeno y cantidad de semilla. Agricultura técnica en México, 33(3): 251-258.

Ayala, A.; Espitia, E.; Rivas, P.; Martínez, G.; Almaguer, G. 2016. Análisis de la cadena del valor de amaranto en México. Agricultura, Sociedad y Desarrollo, (1) 13: 87.

Barreto, J.; Julca, A.; Canto, M.

2015. Sostenibilidad ecológica de la Producción Agropecuaria Tradicional de Carhuaz, Áncash, Perú. Aporte santiaguino, 8 (2): 219-228.

Castro, M.; Fito, P.; Prieto, J.; Andrés, A.; Fito, P.

2012. Study of the puffing process of amaranth seeds by dielectric spectroscopy. Journal of Food Engineering, 110: 298-304.

De Troiani, R.M.; Sánchez, T.; de Ferramola, L.A.

2005. Incidencia de la fertilización en amaranto. Zona semiárida pampeana (Argentina). Revista de la Facultad de Ciencias Agrarias, 37(2): 65-72.

Díaz-Ortega, A.C.; Escalante-Estrada, J. A.; Trinidad-Santos, A.;

Sánchez-García, P.; Mapes-Sánchez, C.; Martínez-Moreno, D. 2004. Rendimiento, eficiencia agronómica del nitrógeno y eficiencia en el uso del agua en amaranto en función del manejo del cultivo. Terra Latinoamericana, 22(1): 109-116.

Estrada, R.

2011. Kiwicha alimento nuestro para el mundo. Instituto Nacional de Innovación Agraria. Cusco, Perú. 41 p.

González, J.; Arroyo, I.L.; Borodanenko, A.; Carballo, C.A.

2005. Efecto de la época de cosecha en las características físicas, químicas y funcionales del amaranto (Amaranthus hypochondriacus). En: VII Congreso Nacional de Ciencias de los Alimentos. Guanajuato, México. Pp. 94-102.

Hernández, E.; García, E.; Ramírez, J.

2014. Caracterización de suelos cultivados con amaranto y algunos aspectos agronómicos de la planta. Revista Mexicana de Ciencias Agrícolas, 5 (3): 421-431.

Jiménez, L.O.; González, M.M.; Bastidas, M.L.; Decker, F.E. 2018. Evaluación del rendimiento de tres sistemas de siembra y dos variedades de amaranto. 2018. (Amaranthus quitensis) y (Amaranthus hypochondriacus). J. Selva Andina Biosph. 2018; 6(2): 65-75.

León, A.; Rosell, C.

2007. De tales harinas, tales panes: granos, harinas y productos de panificación en Iberoamérica. - $1^{\mathrm{a}}$ ed. - Granos andinos. Córdoba, Argentina. 480 p.
Mujica, S.A.; Berti, M.D.

1997. El cultivo del amaranto (Amaranthus spp.): producción, mejoramiento genético y utilización. Oficina Regional de la FAO para América Latina y el Caribe. Santiago, Chile. 145 p.

Ortiz, E.; Argumedo, A.; García, H.; Meza, R.; Bernal, R.; Taboada, O.R.

018. Rendimiento y volumen de expansión de grano de variedades mejoradas de amaranto para valles altos de puebla. Rev. Fitotec. Mex., 41 (3): 291-300.

Paucar, L.; Peñas, E.; Dueñas, M.; Frías, J.; Martínez

Villaluenga, C.

2017. Optimizing germination conditions to enhance the accumulation of bioactive compounds and the antioxidant activity of kiwicha (Amaranthus caudatus) using response surface methodology. Food Science and Technology, 76: 245-252.

Peralta, E.N.; Mazón, A.; Murillo, M.; Rivera, D.; Rodríguez,

L.; Lomas, C.; Monar, C.

2012. Manual Agrícola de Granos Andinos: Chocho, Quinua, Amaranto y Ataco. Cultivos, variedades y costos de producción. Tercera edición. Publicación Miscelánea $\mathrm{N}^{\circ}$ 69. Programa Nacional de Leguminosas y Granos Andinos. Estación Experimental Santa Catalina. INIAP. Quito, Ecuador. 68 pp.

Pinedo, R.; Rodríguez, G.; Valverde, N.

2017. Niveles de fertilización en dos variedades de maíz morado (Zea mayz L.) en la localidad de Canaán-Ayacucho. Aporte Santiaguino, 10 (1): 39-50.

Pinedo, R.; Gómez, L.; Julca, O.

2018. Sostenibilidad de sistemas de producción de quinua (Chenopodium quinoa Willd.) Ecosistemas y Recursos Agropecuarios, 5 (15): 399-409.

Praveen, M.; Usha, J.

2014. Application of NPK and farmyard manure on biomass production of Amaranthus palmeri Wats. Asian Journal of Plant Science and Research, 4 (2): 7-12.

Ramírez, M.; Espitia, E.; Carballo, A.; Zepeda, R.; Vaquera, H.; Córdova, L.

2011. Fertilización y densidad de plantas en variedades de amaranto (Amaranthus hypochondriacus L.). Revista mexicana de ciencias agrícolas, 2 (6): 855-866.

Rojas, W.; Soto, J.L.; Pinto, M.; Jäger, M.; Padulosi, S.

2010. Granos Andinos. Avances, logros y experiencias desarrolladas en quinua, cañahua y amaranto en Bolivia. Bioversity International. Roma, Italia. 185 p.

Romero, C.; Ocampo, J.; Sandoval, E.; Navarro, H.; Franco, O.; Calderón, F.

2017. Fertilización orgánica-mineral del cultivo de amaranto (Amaranthus hypochondriacus L.). Revista Mexicana de Ciencias Agrícolas, 8 (8): 1759-1771. 
Roque, O.J.

2019. Requerimiento térmico de las fases fenológicas de dos variedades de amaranto (Amaranthus caudatus L): precoz y tardía en Ayacucho. Journal of the Selva Andina Biosphere, 7 (1): 18-31.

Soto, J.L.

2010. Tecnología del cultivo de granos andinos. En: Rojas W., Soto J.L., Pinto M., Jäger M., Padulosi S. (editores). Granos Andinos. Avances, logros y experiencias desarrolladas en quinua, cañahua y amaranto en Bolivia. Bioversity International. Roma, Italia. pp. 112-119.
Tapia, M.

2000. Cultivos andinos subexplotados y su aporte a la alimentación. Segunda Edición. Oficina Regional de la FAO para América Latina y el Caribe. Santiago, Chile. $157 \mathrm{p}$.

Topwal, M.A.

2019. Review on Amaranth: Nutraceutical and Virtual Plant for Providing Food Security and Nutrients. Acta Scientific Agriculture, 3(1): 09-15. 
